\title{
Fuzzy Fibonacci and Fuzzy Lucas Numbers with their Properties
}

\author{
Nurettin Irmak and Naime Demirtaş*
}

\begin{abstract}
In this paper, we combine the important concepts which are Fuzzy numbers and Fibonacci, Lucas numbers. We introduce the concepts of Fuzzy Fibonacci and Fuzzy Lucas numbers by this combination. By this motivation, we provide a bridge between the areas Fuzzy sets and number theory. Afterwards, we generalize their well-known properties by the definitions of Fuzzy Fibonacci and Lucas numbers.
\end{abstract}

Keywords: Fuzzy numbers; Fuzzy Fibonacci numbers; Fuzzy Lucas numbers.

AMS Subject Classification (2010): Primary: 11B39 ; Secondary: $03 E 72$.

*Corresponding author

\section{Introduction}

Mathematics is based on exact concepts and there is not vagueness. Since in many other fields such as medicine, engineering, economics and sociology, the notions are vagueness, researchers need to define some new concepts to remove the vagueness. In order to solve the problems about vagueness in these areas, the fuzzy set theory is proposed by Zadeh in 1965. The fuzzy set theory is based on the fuzzy membership function $\mu: A \rightarrow[0,1]$ for any set $A$. By the fuzzy membership function, we can determine the membership grade of an element with respect to a set. A fuzzy set $F$ is described by its membership function $\mu_{F}$. Since fuzzy set theory has a lot of applications in real life, the interest of workings and researchings are increasing in recent years.

In 1978, Dubois and Prade [2] defined fuzzy number which is a fuzzy subset of the real line. A fuzzy number is a generalization of a real number. Because it does not refer to one single value. However it refers to a connected set of possible values, where each possible value has its own membership in $[0,1]$. The concept of fuzzy number is a powerful paradigm for representation of the imprecision in numeric information. Therefore the definitions of arithmetic operations was necessary for fuzzy numbers in order to use the applications to mathematics, engineering, economics etc. The arithmetical structures of fuzzy numbers have been developed in the 1980s and this enabled to design the elements of fuzzy calculus [6, 7]; Dubois and Prade stated the exact analytical fuzzy mathematics and introduced the well-known LR model and the corresponding formulas for the fuzzy operations. See [3, 7] for the basic concepts. More recently, the literature on fuzzy numbers has grown in terms of contributions to fuzzy arithmetic operations and to the use of simple formulas to approximate them; an extensive recent survey and bibliography on fuzzy intervals is in [13]. In general, the arithmetic operations on fuzzy numbers can be approached either by the direct use of the membership function or by the equivalent use of the $\alpha$-cuts representation. By the $\alpha$-cuts approach, it is possible to define a parametric representation of fuzzy numbers that allow a large variety of possible shapes and is very simple to implement, with the advantage of obtaining a much wider family of fuzzy numbers than for standard LR model $[12,19]$. This representation has the relevant advantage of being applied to the same $[0,1]$ interval for all the fuzzy numbers involved in the computations. Gao and Zhang [10] introduced the definitions of the arithmetic operations on triangular fuzzy numbers by the $\alpha$-cuts intervals.

Second important item is Fibonacci and Lucas numbers in this paper. Fibonacci numbers have an important place in mathematics. It is known that the dominant root of the characteristic equation of the Fibonacci sequence is

Received : 22-10-2018, Accepted : 07-10-2019 
$\frac{1+\sqrt{5}}{2} \cong 1.618$ which is called golden ration. One can see this ration in nature, historical artifacts, human bodies etc. There are also a lot of combinatorial identities belongs to Fibonacci numbers. In the issues of the Fibonacci Quarterly, we can find many new facts, applications, and relationships about this numbers. Moreover, we can find the connected sequences such as Lucas numbers, Pell numbers for Fibonacci numbers, too. For more information about Fibonacci and Lucas numbers, we refer to the book [17] for readers.

In the paper of Halıc1 [13], two concepts Fibonacci numbers and quaternions are combined by author. Moreover, well-known identities belongs to Fibonacci and Lucas numbers were obtained for Fibonacci and Lucas quaternions. By motivation of this paper, we think two concepts Fuzzy numbers and Fibonacci, Lucas numbers together. We mean that Fuzzy Fibonacci and Fuzzy Lucas numbers are defined in this paper. Moreover, we investigate the combinatorial identities and relationships both Fuzzy Fibonacci numbers and between Fuzzy Fibonacci and Fuzzy Lucas numbers.

As a result, we think that this study provide a bridge between the areas Fuzzy sets and number theory.

Now, we give the fundamentals about Fuzzy numbers and Fibonacci, Lucas numbers, separately.

\section{Preliminaries}

\subsection{Fuzzy number}

If a fuzzy set is convex and normalized, and its membership function is defined in $\mathbb{R}$ and piecewise continuous, it is called a fuzzy number. There are two types of fuzzy numbers which are triangular fuzzy number and trapezoidal fuzzy number. We focus on triangular fuzzy number in this paper.

Generally, a triangular fuzzy number is represented by three points, two of them are left and right of the interval, the remaining point is a peak point as $\left(a_{1}, a_{2}, a_{3}\right)$ such that $a_{1}, a_{2}, a_{3} \in \mathbb{R}$.

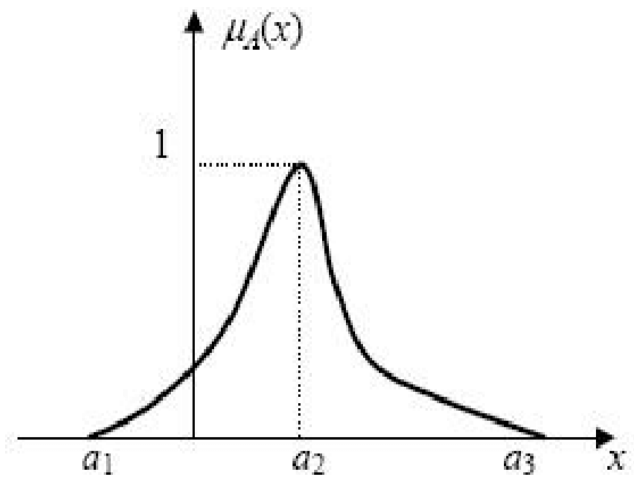

Figure 1. Fuzzy Number $A=\left(a_{1}, a_{2}, a_{3}\right)$

A triangular fuzzy number $A=\left(a_{1}, a_{2}, a_{3}\right)$ can be represented by $\alpha$-cut operation which we denote $A^{\alpha}$. To convert a triangular fuzzy number to $\alpha$-cut interval, we follow that

$$
A^{\alpha}=\left[a_{1}^{\alpha}, a_{3}^{\alpha}\right]=\left[a_{1}+\alpha\left(a_{2}-a_{1}\right), a_{3}-\alpha\left(a_{3}-a_{2}\right)\right]
$$

where $\alpha \in[0,1]$ and $a_{i}^{\alpha} \in \mathbb{R}$ for $i=1,3$.

Assume that $A^{\alpha}=\left[a_{1}^{\alpha}, a_{3}^{\alpha}\right]$ and $B^{\alpha}=\left[b_{1}^{\alpha}, b_{3}^{\alpha}\right]$ are two $\alpha$-cut intervals. The arithmetic operations of the $\alpha$-cut intervals are followings.

1. Addition

$$
A^{\alpha}+B^{\alpha}=\left[a_{1}^{\alpha}+b_{1}^{\alpha}, a_{3}^{\alpha}+b_{3}^{\alpha}\right]
$$

2. Subtraction

$$
A^{\alpha}-B^{\alpha}=\left[a_{1}^{\alpha}-b_{1}^{\alpha}, a_{3}^{\alpha}-b_{3}^{\alpha}\right]
$$

\section{Multiplication}

$$
A^{\alpha} \cdot B^{\alpha}=\left[\min \left\{a_{1}^{\alpha} b_{1}^{\alpha}, a_{1}^{\alpha} b_{3}^{\alpha}, a_{3}^{\alpha} b_{1}^{\alpha}, a_{3}^{\alpha} b_{3}^{\alpha}\right\}, \max \left\{a_{1}^{\alpha} b_{1}^{\alpha}, a_{1}^{\alpha} b_{3}^{\alpha}, a_{3}^{\alpha} b_{1}^{\alpha}, a_{3}^{\alpha} b_{3}^{\alpha}\right\}\right]
$$


4. Division

$$
A^{\alpha} / B^{\alpha}=\left[\min \left\{a_{1}^{\alpha} / b_{1}^{\alpha}, a_{1}^{\alpha} / b_{3}^{\alpha}, a_{3}^{\alpha} / b_{1}^{\alpha}, a_{3}^{\alpha} / b_{3}^{\alpha}\right\}, \max \left\{a_{1}^{\alpha} / b_{1}^{\alpha}, a_{1}^{\alpha} / b_{3}^{\alpha}, a_{3}^{\alpha} / b_{1}^{\alpha}, a_{3}^{\alpha} / b_{3}^{\alpha}\right\}\right]
$$

such that $0 \notin\left\{b_{1}^{\alpha}, b_{3}^{\alpha}\right\}$.

\section{Multiplication with scaler number}

$$
k \cdot A^{\alpha}=\left[\min \left\{k a_{1}^{\alpha}, k a_{3}^{\alpha}\right\}, \max \left\{k a_{1}^{\alpha}, k a_{3}^{\alpha}\right\}\right]
$$

where $k \in \mathbb{R}$.

\subsection{Fibonacci and Lucas numbers}

Assume that $F_{n}$ denotes the $n$th Fibonacci number which defining by the following relation. For $n \geq 2$,

$$
F_{n}=F_{n-1}+F_{n-2}
$$

with the initial conditions $F_{0}=0$ and $F_{1}=1$. A few Fibonacci numbers are,

$$
0,1,1,2,3,5,8,13,21, \ldots
$$

Let $L_{n}$ denotes the $n$th Lucas numbers. Lucas numbers are known the member of the associated sequence of Fibonacci numbers. That is, Lucas numbers satisfies the same recurrence relation with the different initial conditions of Fibonacci numbers.

$$
L_{n}=L_{n-1}+L_{n-2}
$$

together with $L_{0}=2$ and $L_{1}=1$

\subsection{Fuzzy Fibonacci and Lucas Numbers}

In this section, we define the Fuzzy Fibonacci and Fuzzy Lucas numbers by using Fibonacci and Lucas numbers.

Definition 2.1. Let $\left\{F_{n}\right\}$ be Fibonacci sequence. For $n \geq 0$ integer, Fuzzy Fibonacci numbers are defined as

$$
F_{r n+l}^{\alpha}=\left[F_{r(n-1)+l}+\alpha\left(F_{r n+l}-F_{r(n-1)+l}\right), F_{r(n+1)+l}-\alpha\left(F_{r(n+1)+l}-F_{r n+l}\right)\right]
$$

where $r, l \in \mathbb{Z}^{+}$and $\alpha \in[0,1]$.

When we take $r=1$ and $l=0$, several Fuzzy Fibonacci numbers are following;

$$
F_{0}^{\alpha}=[1-\alpha, 1+\alpha], F_{1}^{\alpha}=[\alpha, 1], F_{2}^{\alpha}=[1,2+\alpha], F_{3}^{\alpha}=[1+\alpha, 3+\alpha], \ldots
$$

Since Fuzzy Fibonacci numbers include Fibonacci numbers, then Fuzzy Fibonacci numbers satisfy the similar recurrence relation (2.6) with initial conditions $F_{0}^{\alpha}=[1-\alpha, 1+\alpha], F_{1}^{\alpha}=[\alpha, 1]$. That is

$$
F_{n}^{\alpha}=F_{n-1}^{\alpha}+F_{n-2}^{\alpha}
$$

for $n \geq 2$.

Definition 2.2. Let $\left\{L_{n}\right\}$ be Lucas sequence. For $n \geq 0$ integer, Fuzzy Lucas numbers are defined as

$$
L_{r n+l}^{\alpha}=\left[L_{r(n-1)+l}+\alpha\left(L_{r n+l}-L_{r(n-1)+l}\right), L_{r(n+1)+l}-\alpha\left(L_{r(n+1)+l}-L_{r n+l}\right)\right]
$$

where $r, l \in \mathbb{Z}^{+}$and $\alpha \in[0,1]$.

When we take $r=1$ and $l=0$, several Fuzzy Lucas numbers are following;

$$
L_{1}^{\alpha}=[2-\alpha, 3-2 \alpha], L_{2}^{\alpha}=[1+2 \alpha, 4-\alpha], L_{3}^{\alpha}=[3+\alpha, 7-3 \alpha], \ldots
$$

Together with initial conditions $L_{0}^{\alpha}=[-1+3 \alpha, 1+\alpha], L_{1}^{\alpha}=[2-\alpha, 3-2 \alpha]$, Fuzzy Lucas numbers satisfy recurrence relation. That is

$$
L_{n}^{\alpha}=L_{n-1}^{\alpha}+L_{n-2}^{\alpha}
$$

for $n \geq 2$. 


\section{Identities of Fuzzy Fibonacci and Fuzzy Lucas Numbers}

In this part, we generalize some well-known identities of Fibonacci and Lucas numbers to Fuzzy Fibonacci and Fuzzy Lucas numbers. We see that although some of the properties remain same, the others change. Now we give them.

Theorem 3.1. $F_{n}^{\alpha}$ and $L_{n}^{\alpha}$ be nth Fuzzy Fibonacci and Lucas numbers such that $n \geq 0$, respectively. Then

$$
F_{n+2}^{\alpha}-F_{n-2}^{\alpha}=L_{n}^{\alpha}
$$

holds.

Proof. By the Definition 2.1. and (2.2), we have

$$
\begin{aligned}
F_{n+2}^{\alpha}-F_{n-2}^{\alpha}= & {\left[F_{n+1}+\alpha F_{n}, F_{n+3}-\alpha F_{n+1}\right] } \\
& -\left[F_{n-3}+\alpha F_{n-4}, F_{n-1}-\alpha F_{n-3}\right] \\
= & {\left[F_{n+1}-F_{n-3}+\alpha\left(F_{n}-F_{n-4}\right)\right.} \\
& \left.F_{n+3}-F_{n-1}-\alpha\left(F_{n+1}-F_{n-3}\right)\right] .
\end{aligned}
$$

Since $F_{n+2}-F_{n-2}=L_{n}$, then we get together with the Definition 2.2.

$$
\begin{aligned}
F_{n+2}^{\alpha}-F_{n-2}^{\alpha} & =\left[L_{n-1}+\alpha L_{n-2}, L_{n+1}-\alpha L_{n-1}\right] \\
& =L_{n}^{\alpha} .
\end{aligned}
$$

Theorem 3.2. Let $n \geq 0$ integer. Assume that $F_{n}^{\alpha}$ and $L_{n}^{\alpha}$ be $n$th Fuzzy Fibonacci and Lucas numbers, respectively. Then we have

(a) $2 F_{n+2}^{\alpha}=3 F_{n}^{\alpha}+L_{n}^{\alpha}$

(b) $2 L_{n+2}^{\alpha}=5 F_{n}^{\alpha}+3 L_{n}^{\alpha}$

(c) $5 F_{n}^{\alpha}=L_{n+1}^{\alpha}+L_{n-1}^{\alpha}$

(d) $2 F_{n+1}^{\alpha}=F_{n}^{\alpha}+L_{n}^{\alpha}$

(e) $2 L_{n+1}^{\alpha}=5 F_{n}^{\alpha}+L_{n}^{\alpha}$

(f) $F_{n} F_{m-1}^{\alpha}+F_{n+1} F_{m}^{\alpha}=F_{m+n}^{\alpha}$

(g) $L_{n}^{\alpha}=F_{n-1}^{\alpha}+F_{n+1}^{\alpha}$

Proof. Since the method of the proof is same as the proof of Theorem 3.1, we omit this proof to cut the unnecessary repetition.

In the sequel, we give identities including product of Fuzzy Fibonacci and Lucas numbers.

Theorem 3.3. Let $m, n$ be non-negative integers and $F_{n}^{\alpha}$ be $n$th Fuzzy Fibonacci number. Then

$$
\begin{aligned}
F_{m}^{\alpha} F_{n-m+1}^{\alpha}+F_{m-1}^{\alpha} F_{n-m}^{\alpha} & \cong\left(F_{n-2}, F_{n}, F_{n+2}\right) \\
& =\left[F_{n-2}+\alpha\left(F_{n}-F_{n-2}\right), F_{n+2}-\alpha\left(F_{n+2}-F_{n}\right)\right]
\end{aligned}
$$

holds. 
Proof. Using the Definition 2.1. and arithmetic operations for fuzzy numbers, we get

$$
\begin{aligned}
F_{m}^{\alpha} F_{n-m+1}^{\alpha}+F_{m-1}^{\alpha} F_{n-m}^{\alpha}= & {\left[F_{m-1}+\alpha F_{m-2}, F_{m+1}-\alpha F_{m-1}\right] } \\
& \times\left[F_{n-m}+\alpha F_{n-m-1}, F_{n-m+2}-\alpha F_{n-m}\right] \\
& +\left[F_{m-2}+\alpha F_{m-3}, F_{m}-\alpha F_{m-2}\right] \\
& \times\left[F_{n-m-1}+\alpha F_{n-m-2}, F_{n-m+1}-\alpha F_{n-m-1}\right] \\
= & {\left[\left(F_{m-1}+\alpha F_{m-2}\right)\left(F_{n-m}+\alpha F_{n-m-1}\right),\right.} \\
& \left.\left(F_{m+1}-\alpha F_{m-1}\right)\left(F_{n-m+2}-\alpha F_{n-m}\right)\right] \\
& +\left[\left(F_{m-2}+\alpha F_{m-3}\right)\left(F_{n-m-1}+\alpha F_{n-m-2}\right),\right. \\
& \left.\left(F_{m}-\alpha F_{m-2}\right)\left(F_{n-m+1}-\alpha F_{n-m-1}\right)\right] \\
= & {\left[F_{m-1} F_{n-m}+F_{m-2} F_{n-m-1}+\right.} \\
& \alpha\left(F_{m-1} F_{n-m-1}+F_{m-2} F_{n-m}\right. \\
& \left.+F_{m-2} F_{n-m-2}+F_{m-3} F_{n-m-1}\right) \\
& +\alpha^{2}\left(F_{m-2} F_{n-m-1}+F_{m-3} F_{n-m-2}\right) \\
& F_{m+1} F_{n-m+2}+F_{m} F_{n-m+1}- \\
& \alpha\left(F_{m-1} F_{n-m+2}+F_{m+1} F_{n-m}\right. \\
& \left.+F_{m} F_{n-m-1}+F_{m-2} F_{n-m+1}\right) \\
& \left.+\alpha^{2}\left(F_{m-1} F_{n-m}+F_{m-2} F_{n-m-1}\right)\right]
\end{aligned}
$$

Since $F_{m} F_{n-m+1}+F_{m-1} F_{n-m}=F_{n}$ holds for non-negative integers $n$ and $m$, we obtain that

$$
\begin{aligned}
F_{m}^{\alpha} F_{n-m+1}^{\alpha}+F_{m-1}^{\alpha} F_{n-m}^{\alpha}= & {\left[F_{n-2}+2 \alpha F_{n-3}+\alpha^{2} F_{n-4},\right.} \\
& \left.F_{n+2}-2 \alpha F_{n}+\alpha^{2} F_{n-2}\right] .
\end{aligned}
$$

We put $\alpha=0$ and $\alpha=1$ in (3.1) in order to convert this Fuzzy number to $\alpha$-cut interval. If we use the formula (2.1), then we get $a_{1}=F_{n-2}, a_{2}=F_{n}$ and $a_{3}=F_{n+2}$.

$$
\begin{aligned}
F_{m}^{\alpha} F_{n-m+1}^{\alpha}+F_{m-1}^{\alpha} F_{n-m}^{\alpha} & \cong\left[F_{n-2}+\alpha\left(F_{n}-F_{n-2}\right), F_{n+2}-\alpha\left(F_{n+2}-F_{n}\right)\right] \\
& =\left(F_{n-2}, F_{n}, F_{n+2}\right)
\end{aligned}
$$

is obtained by the formula (2.1).

The identities in the following theorem can be proven by using the method in the proof of the Theorem 3.3. Therefore, we give them without their proof.

Theorem 3.4. For non-negative integer $n, m$ and $\alpha \in[0,1]$, the following identities hold

(a) $\left(F_{n}^{\alpha}\right)^{2}+\left(F_{n+1}^{\alpha}\right)^{2} \cong F_{2 n+1}^{\alpha}$

(b) $F_{2 n}^{\alpha} \cong F_{n}^{\alpha} L_{n}^{\alpha}$

(c) $2 L_{2 n} \cong 5\left(F_{n}^{\alpha}\right)^{2}+\left(L_{n}^{\alpha}\right)^{2}$

(d) $\left(F_{n+2 k}^{\alpha}\right)^{2}-\left(F_{n}^{\alpha}\right)^{2} \cong F_{2 k} F_{2 n+2 k}^{\alpha}$

(e) $F_{n}^{\alpha} F_{m-1}^{\alpha}+F_{n+1}^{\alpha} F_{m}^{\alpha} \cong\left(F_{n+m-2}, F_{n+m}, F_{n+m+2}\right)$

(f) $5 F_{m}^{\alpha} F_{n}^{\alpha}+L_{m}^{\alpha} L_{n}^{\alpha} \cong 2 L_{n+m}^{\alpha}$.

One can see easily that the well-known Fibonacci and Lucas identities are obtained when we put $\alpha=1$ in all of the these identities.

Remark 3.1.

1. We observe that if we take the coefficients $F_{n}, F_{n+1}$ or $F_{m-1}, F_{m}$ as Fibonacci numbers in (f) of Theorem 2, then the identity does not change. However, if we replace the coefficients $F_{n}^{\alpha}, F_{n+1}^{\alpha}\left(o r F_{m-1}^{\alpha}, F_{m}^{\alpha}\right)$ as Fuzzy Fibonacci numbers in (e) of Theorem 4, then one can see that the identity changes.

2. Addition and subtraction of Fuzzy Fibonacci and Fuzzy Lucas numbers are a Fuzzy number. This situation is also valid for multiplication with a scaler of a Fuzzy Fibonacci and Fuzzy Lucas number. But, multiplications of Fuzzy Fibonacci and Fuzzy Lucas numbers do not fit the definition of Fuzzy number since we obtain second order polynomial in the components of $\alpha$-cut interval. In order to remove the terms $\alpha^{2}$, we put $\alpha=0$ and $\alpha=1$ in the second order polynomials, respectively. Therefore, we have approximate values in Theorem 3.3 and Theorem 3.4. 
Theorem 3.5. For non-negative integer $n$, we have the followings,

(a) $\sum_{n=0}^{k} F_{n}^{\alpha}=F_{k+2}^{\alpha}-F_{1}^{\alpha}$

(b) $\sum_{n=0}^{k} F_{2 n}^{\alpha}=F_{2 k+1}^{\alpha}-F_{1}^{\alpha}$

(c) $\sum_{n=0}^{k-1} F_{2 n+1}^{\alpha}=F_{2 k}^{\alpha}$

Proof. (a) By the recurrence relation of Fuzzy Fibonacci sequence, we have

$$
F_{n}^{\alpha}=F_{n+2}^{\alpha}-F_{n+1}^{\alpha} .
$$

So,

$$
\sum_{n=0}^{k} F_{n}^{\alpha}=\sum_{n=0}^{k} F_{n+2}^{\alpha}-F_{n+1}^{\alpha}
$$

By telescoping sum,

$$
\sum_{n=0}^{k} F_{n}^{\alpha}=F_{k+2}^{\alpha}-F_{1}^{\alpha}
$$

follows as claimed.

(b) By the definition of the Fuzzy Fibonacci numbers, we get,

$$
\begin{aligned}
\sum_{n=0}^{k} F_{2 n}^{\alpha} & =\sum_{n=0}^{k}\left[F_{2(n-1)}+\alpha F_{2 n-1}, F_{2 n+2}-\alpha F_{2 n+1}\right] \\
& =\left[\sum_{n=0}^{k} F_{2(n-1)}+\alpha \sum_{n=0}^{k} F_{2 n-1}, \sum_{n=0}^{k} F_{2 n+2}-\alpha \sum_{n=0}^{k} F_{2 n+1}\right] \\
& =\left[F_{2 k-1}+\alpha\left(F_{2 k}-1\right), F_{2 k+3}-1-\alpha F_{2 k+3}\right] \\
& =\left[F_{2 k-1}+\alpha F_{2 k}, F_{2 k+3}-\alpha F_{2 k+3}\right]-[\alpha, 1] \\
& =F_{2 k+1}^{\alpha}-F_{1}^{\alpha}
\end{aligned}
$$

So, we obtain the claimed result. The (c) can be proven by similarly. Therefore, we give it without proofs.

\section{Conclusion}

A fuzzy number is simply an ordinary number whose exact value is somewhat uncertain. Fuzzy numbers allow us to make the mathematical model of vagueness and are used in statistics, computer programming, engineering and experimental science.

In this paper we define Fuzzy Fibonacci and Fuzzy Lucas numbers. Afterwards, we give several combinatorial identities including these numbers. By the means of the these concepts, we combine two important areas which are linear recurrences and fuzzy set theory. We believe that this study can be applied to other identities or carried other areas of linear recurrences in future.

\section{References}

[1] Bandemer, H., Mathematics of Uncertainty: Ideas, Methods, Application Problems. Springer, New York, 2006.

[2] Dubois, D. and Prade, H., Operations on Fuzzy Numbers,Int. J. Systems Sci., 9-6, 613-626, 1978.

[3] Dubois D. and Prade H., Towards fuzzy differential calculus. Fuzzy Sets Syst. 8 (1982) 1-17(I), 105-116(II), 225-234(III).

[4] Diamond P. and Kloeden. P., Metric Spaces of Fuzzy Sets. World Scientific, Singapore, 1994.

[5] Dubois D. and Prade H., Fuzzy Sets and Systems: Theory and Applications. Academic Press, New York, 1980.

[6] Dubois D. and Prade H., Ranking fuzzy numbers in a setting of possibility theory. Inf. Sci. 30 (1983) 183-224. 
[7] Dubois D. and Prade H., Possibility Theory. An Approach to Computerized Processing of Uncertainty. Plenum Press, New York, 1988.

[8] Dubois D. and Prade H., (eds). Fundamentals of Fuzzy Sets, The Handbooks of Fuzzy Sets Series. Kluwer, Boston, 2000.

[9] Dubois D., Kerre E., Mesiar R. and Prade H., Fuzzy interval analysis. In: D. Dubois and H. Prade (eds), Fundamentals of Fuzzy Sets, The Handbooks of Fuzzy Sets Series. Kluwer, Boston, 2000, pp. 483-581.

[10] Gao, S., Zhang, Z. and Cao, C., Multiplication Operation on Fuzzy Numbers,Journal of Software, 4-4, 331-338, 2009.

[11] Goetschel R. and Voxman W., Elementary fuzzy calculus. Fuzzy Sets Syst. 18 (1986) 31-43.

[12] Guerra M.L. and Stefanini L., Approximate fuzzy arithmetic operations using monotonic interpolations. Fuzzy Sets Syst. 150 (2005) 5-33.

[13] Halıc1, S., On Fibonacci Quaterions, Adv. Appl. Clifford Algebras, 12 (2012), 321-327.

[14] Kaufmann, A. and Gupta, M.M., Introduction to Fuzzy Arithmetic - Theory and Applications. Van Nostrand Reinhold, New York, 1985.

[15] Klir, G.J., Uncertainty Analysis in Engineering and Science, Kluwer, Dordrecht, 1997.

[16] Klir, G.J. and Yuan B., Fuzzy Sets and Fuzzy Logic: Theory and Applications. Prentice Hall, Englewood Cliffs, NJ, 1995.

[17] Koshy, T., Fibonacci and Lucas numbers with their identities, Wiley, New York, 2001.

[18] Stefanini L., Sorini L. and Guerra M.L., Parametric representation of fuzzy numbers and application to fuzzy calculus. Fuzzy Sets Syst. 157 (2006) 2423-2455

[19] Stefanini L. and Sorini L., and Guerra M.L., Simulation of fuzzy dynamical systems using the LU-representation of fuzzy numbers. Chaos Solitons Fractals 29(3) (2006) 638-652.

[20] Zimmermann H.-J., Fuzzy Set Theory and Its Applications, 4th edn. Kluwer, Dordrecht, 2001.

[21] Zadeh, L. A., Fuzzy Sets, Information and Control, 8-3, 338-353, 1965.

\section{Affiliations}

\section{NURETTIN IRMAK}

AdDress: Niğde Ömer Halisdemir University, Art and Science Faculty, Mathematics Department, Niğde, Turkey E-MAIL: irmaknurettin@gmail.com, nirmak@ohu.edu.tr

ORCID ID: 0000-0003-0409-4342

NAIME DEMIRTAŞ

AdDress: Mersin University, Art and Science Faculty, Mathematics Department, Mersin, Turkey.

E-MAIL: naimedemirtas@mersin.edu.tr

ORCID ID: 0000-0003-4137-4810 